This item was submitted to Loughborough's Research Repository by the author.

Items in Figshare are protected by copyright, with all rights reserved, unless otherwise indicated.

\title{
Analysis of creep behavior of polypropylene fibers
}

PLEASE CITE THE PUBLISHED VERSION

http://www.ttp.net/1660-9336.html

\section{PUBLISHER}

(c) Trans Tech Publications Inc.

\section{VERSION}

AM (Accepted Manuscript)

\section{LICENCE}

CC BY-NC-ND 4.0

\section{REPOSITORY RECORD}

Sabuncuoglu, Baris, Memis Acar, and Vadim V. Silberschmidt. 2019. "Analysis of Creep Behavior of Polypropylene Fibers". figshare. https://hdl.handle.net/2134/8728. 
This item was submitted to Loughborough's Institutional Repository (https://dspace.lboro.ac.uk/) by the author and is made available under the following Creative Commons Licence conditions.

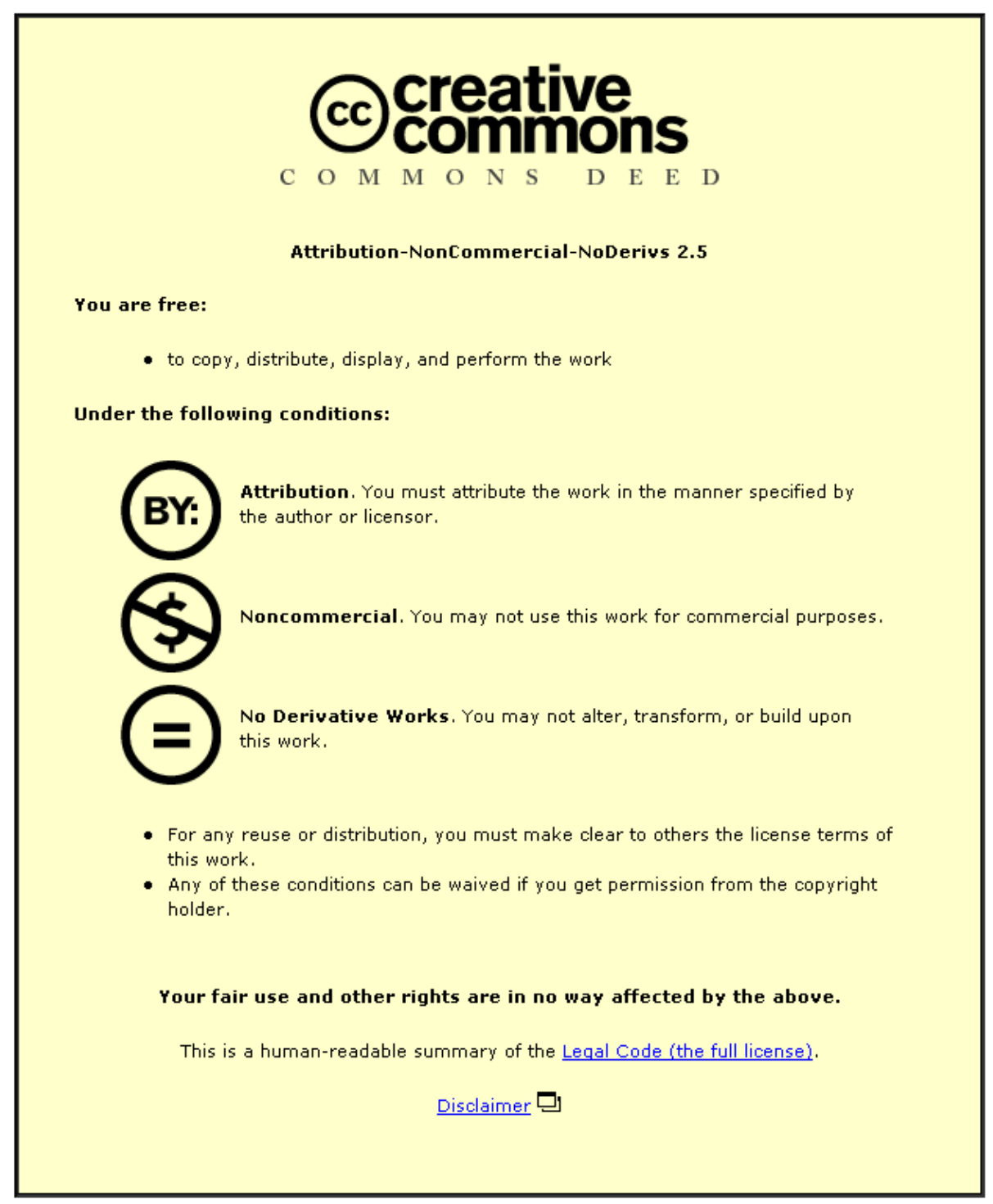

For the full text of this licence, please go to: http://creativecommons.org/licenses/by-nc-nd/2.5/ 


\title{
Analysis of Creep Behavior of Polypropylene Fibers \\ Baris Sabuncuoglu ${ }^{\mathrm{a}}$, M. Acar ${ }^{\mathrm{b}}$ and Vadim V. Silberschmidt ${ }^{\mathrm{c}}$ \\ Wolfson School of Mechanical and Manufacturing Engineering, Loughborough University, Loughborough, Leicestershire, LE11 3TU, UK \\ âB.Sabuncuoglu@lboro.ac.uk, M.Acar@lboro.ac.uk, 'V.Silberschmidt@lboro.ac.uk
}

Keywords: Viscoelasticity, creep, finite element method, polypropylene, nonwoven

\begin{abstract}
Time and stress-dependent viscoelastic properties of polypropylene fibers are determined by means of a series of creep tests and data analysis technique. Relaxation tests are carried out to verify and update the suggested model. The tests are performed for single polypropylene fibers that form a thermally bonded low-density nonwoven. The obtained properties are implemented into the finite element analysis software MSC. Marc - Mentat. Tensile tests and simulations are performed in order to demonstrate suitability of the developed creep model. The obtained results are discussed and further recommendations are given.
\end{abstract}

\section{Introduction}

The focus of the paper is on creep of polypropylene fibers, used to manufacture nonwoven fabrics. One of the methods to determine viscoelastic properties of materials is analysis of their creep behaviour. In a creep test, a specimen is stretched rapidly to a certain constant stress level, and a change in its extension with time is recorded at this stress level. This method allows determination of the effects of stress and time separately on the material's viscous behaviour. It is important to mention that the time-dependent deformation mechanism of creep mathematically correlates with stress relaxation [1]. Thus, it is possible to determine the viscoelastic features of materials by performing creep tests.

The study presented here differs from those in published works in several aspects. First, the mechanical properties are determined in tests conducted for a short period of time. Creep tests are generally performed for long times, at least one hour [2-4]. The primary stage of creep is often ignored in the analysis, and the respective studies concentrate on the secondary region of creep, in which the logarithm of the creep curve is a straight line. In addition, this study links the viscoelastic material property determination with inputs required for finite element simulations; this part is often missing in the literature. Finite-element software MSC. Marc Mentat 2010 is used in this study.

\section{Methodology}

The aim of this study is to develop a procedure to determine the viscoelastic properties of polymeric fibers to be simulated with finite element programs. A general outline of the study is presented in Fig. 1. First, the creep tests are performed with different initial stress levels. The data obtained from the tests are analyzed to determine the stress- and time-dependency of creep, and the obtained data are implemented into the finite element software. Then, the model is verified and updated by relaxation tests and simulations. Finally, tension tests with low strain rates are performed to present the applicability of the developed creep model.

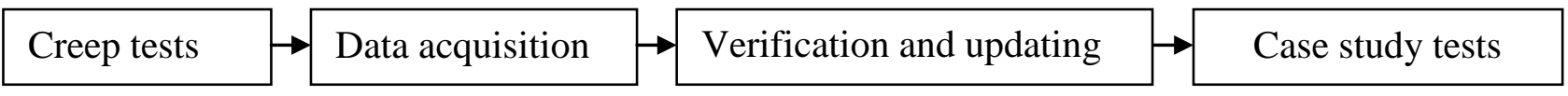

Fig. 1. Procedure used in this study 


\section{Test Samples and Configuration}

The test samples used in this study are single mono-component polypropylene fibers used in a lowdensity thermally bonded nonwoven material. This kind of nonwovens is manufactured by feeding the web of fibers through a heated calendar. The obtained pattern is created by two rolls pressed against each other [5]. One of these two rolls has some engraving on it, and the fibers are attached to each other by partially melting them in those regions. During the manufacturing process of this kind of nonwovens, the fibers are passed through a drawing operation to increase their strength and crimpled to entangle each other. Thus, generally it is not possible to use the material properties of polypropylene manufactured from another batch. In our study, the fiber samples are extracted from a free side of the nonwoven. In these regions, there exist some loose fibers not melted and attached properly; they could be picked without applying a preload on them. The fibers have a diameter of approximately $20 \mu \mathrm{m}$ and lengths varying between 20 and $30 \mathrm{~mm}$ [6]. Before the tests, fiber samples are fixed in the jaws held by the Instron MicroTester test machine. A very sensitive $5 \mathrm{~N}$ load cell is used to determine the load applied on the fiber samples.

\section{Creep Tests}

The tests are performed by stretching the fiber samples up to certain engineering stress levels and holding the achieved stress level constant. The maximum engineering stress level is determined to be $80 \mathrm{MPa}$, which is around the material's yield point [4] determined previously by tensile tests. Tests are performed with $10 \mathrm{MPa}$ increments of stress up to the determined maximum engineering stress level. The test results are shown in Fig. 2. As it can be seen from Fig. 2a, because of its displacement-control feature, the machine switches on and off the crosshead displacement in order to stay close to the pre-defined stress introducing some fluctuations in its level. Respective changes in cross-head displacement are shown in Fig. 2b, which reveals a significant creep component in the deformation behaviour of the material. As it can be seen, the creep behaviour changes with time and the stress applied on the specimen.

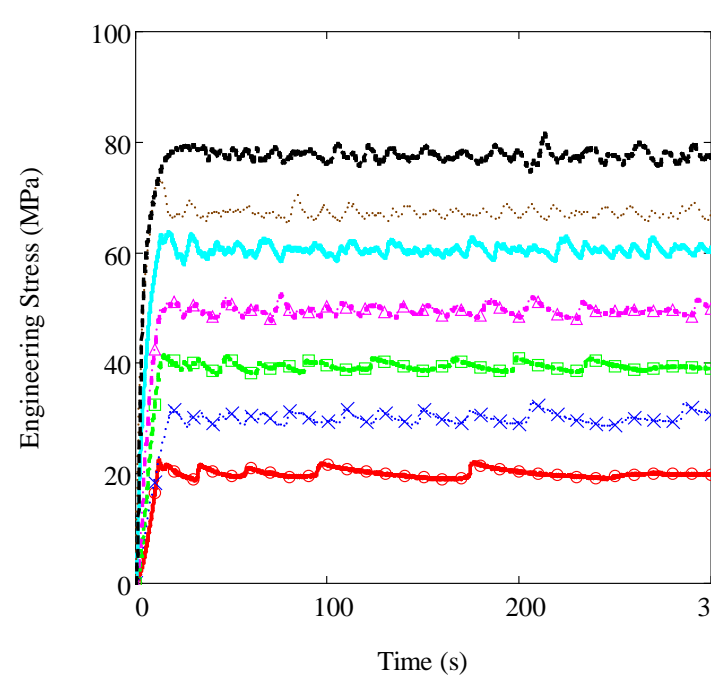

(a)

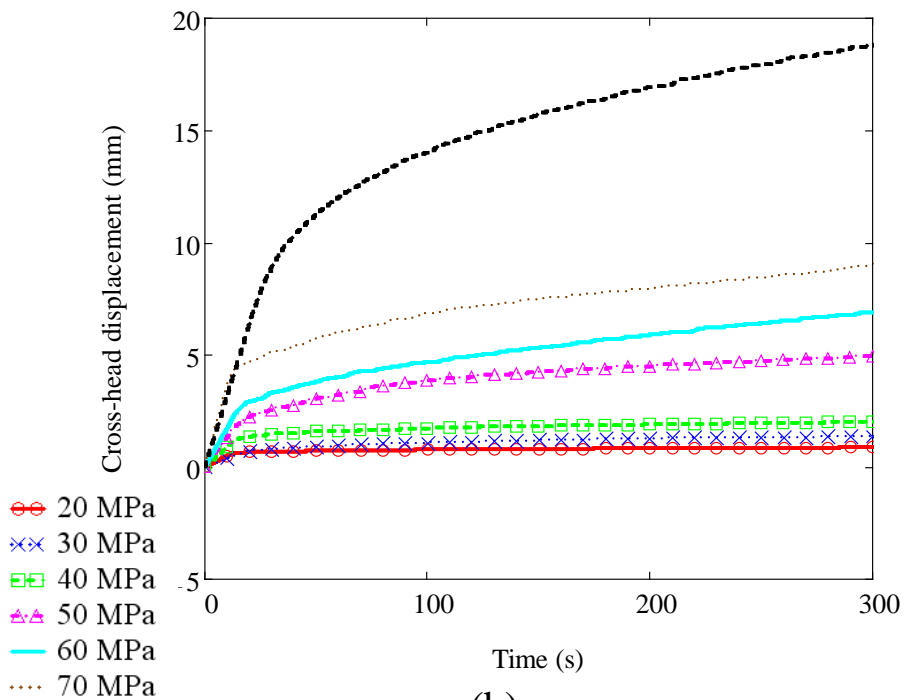

(b)

Fig. 2. Creep test results: (a) Evolution of engineering stress values; (b) Crosshead displacement with time for different stress values

\section{Data Acquisition}

In the performed tests, initially engineering stress and strain acting on the samples are converted to true stress and strain values because of extensive deformations observed during the tests. The data are analyzed after the time when the constant stress levels are reached. 
Creep strain versus time and stress. The true strain - time plots for each levels of stress are plotted, and it was determined that the values can be approximated with the following logarithmic function:

$$
F I T(x)_{i}=C 1_{i}\left(\ln (x)+C 2_{i}\right)+C 3_{i},
$$

where $i$ shows different stress levels; $C 1, C 2$ and $C 2$ are fitting coefficients, which are different for each test. Similarly, creep strain curves are plotted with respect to change in true stress values. The following power equation is determined to be the best fit:

$$
F I T(x)_{i}=C 1_{i} x_{i}^{C 2_{i}}+C 3_{i},
$$

As every fitting operation according to a variable modifies the fit with respect to the other, further iterations are carried out until no significant change is observed. The obtained values are extrapolated to the initial stage, at which the constant stress levels have not been achieved yet.

Creep strain rate. Creep strain-rate values are calculated by dividing the creep strain with time increments. The curve in Fig. 3 is obtained for the change of creep strain rate with respect to time and stress

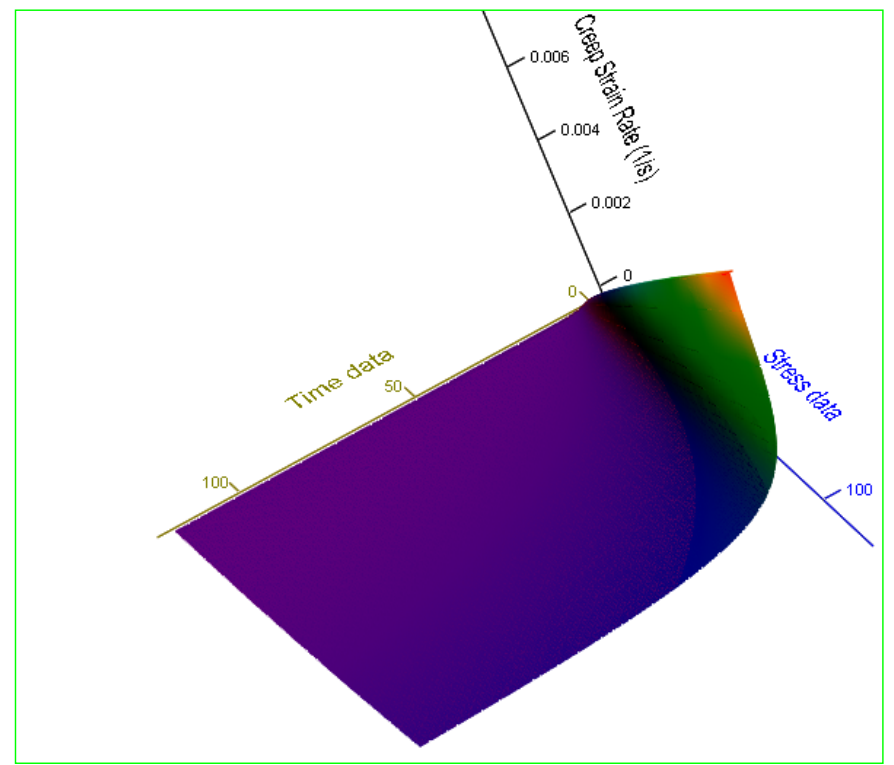

Fig. 3. Creep strain rate for various stress and time levels

As it can be seen, the creep strain rate is higher during the initial stages of loading and decreases with time as was also observed for other types of fibers used in nonwovens [7]. The rate increases significantly with the stress values as found for many polymers [4].

\section{Verification and Updating}

In order to verify the model at the end of the study, some relaxation tests with different stress levels are performed and compared with the simulations employing the creep data. Numerical models used for these studies are composed of one truss element with the same gage length as that measured during the tests. The exact displacement profile of the crosshead in the test is also applied in the simulated model. A nonlinear stress vs. strain curve, obtained from a rapid tension test $(15 \mathrm{~mm} / \mathrm{s})$, is introduced to the model with the creep strain-rate data obtained before. Fig. 4a shows one of those test data compared with the simulations. Compared to the tests, less relaxation is observed during the initial stages of loading in the simulations. It was determined that the extrapolation of determined curves is not enough to represent the initial stages of viscoelastic behaviour. Thus, it was decided to modify the coefficients for the initial stage. After several iterations, it was found that 
doubling the coefficient $\mathrm{C} 2$ in equation 1 during the first 4 seconds provided similar behaviours in tests and simulations (Fig. 4b).

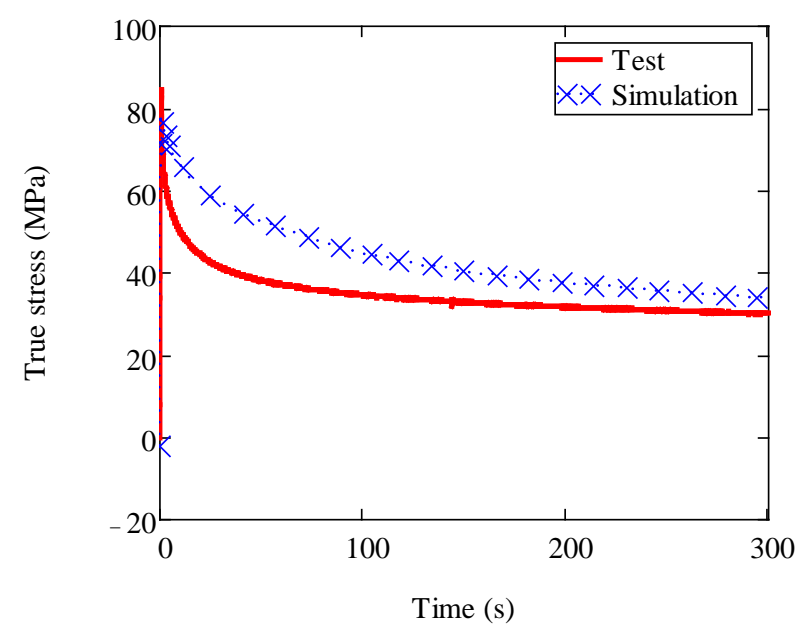

(a)

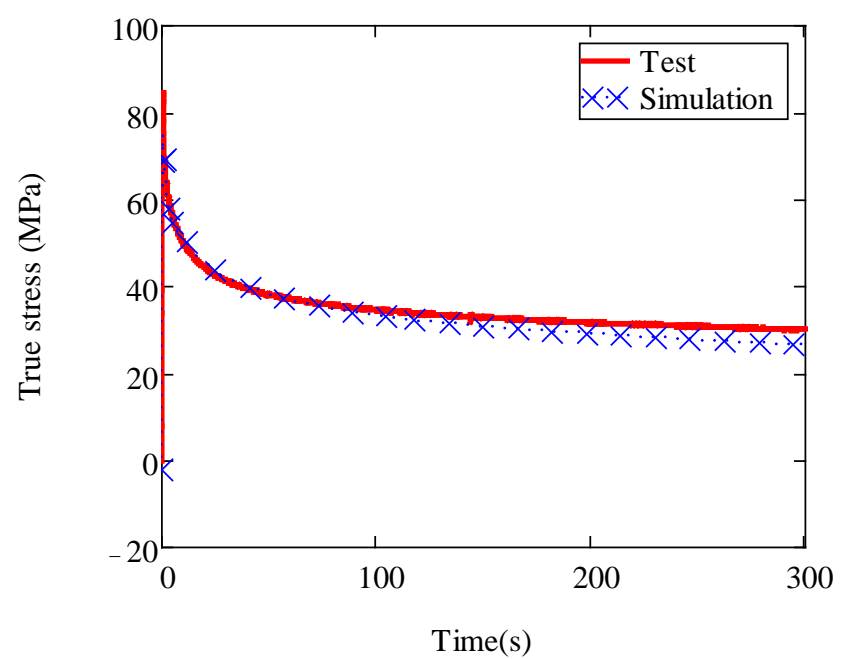

(b)

Fig. 4. Relaxation test results from initial engineering stress level of $75 \mathrm{MPa}$ : (a) original model; (b) updated model

\section{Tensile Case Study Tests}

Tensile tests with various loading rates are performed and simulated to study the effect of implementation of determined creep properties. The analyses are carried out with and without implementation of creep behavior. The test and simulation with the true strain rate of $0.0011 / \mathrm{s}$ are shown in Fig. 5. In Fig. 5a, hypoelastic (nonlinear elastic) stress vs. strain curve is given; the figure demonstrates the importance of implementation of creep behavior in order to achieve realistic results for this test speed. In Fig. 5b, the deformation behavior in the plastic region is implemented with linear elastic material property as the software does not have the option of implementing nonlinear elastic behavior with plasticity. The data for undetermined stress and time values are extrapolated by the software. It is observed that the creep rate becomes too high for the plastic region. Another tensile test with the same speed $(0.0011 / \mathrm{s})$ and with the true strain rate of $0.011 / \mathrm{s}$ are performed and simulated with nonlinear stress-strain curves (Fig. 6). In Fig. 6a, it can be seen that the simulations with and without creep give similar results with a test speed of $0.011 / \mathrm{s}$ true strain rate. In Fig. 6b, there is a large difference between results of the test and simulations even for the simulation with creep properties implemented. There is a slight curvature during the initial stage of loading during the test resulting in a shift of experimental data to higher strain levels. This is attributed mainly to the crimp on fibers. Fig. 7 shows the sample images taken with a microscope. Apparently, there is a crimp on the sample. Hence, in all the tests, first, the fibers are extended until some load is measured before starting the tests in order to avoid the effect of crimp; this procedure fully straighten them. However, some samples still have a crimpled structure, which cannot be seen with a naked eye, even after some load has already been observed. Thus, in the first stages, a different material behavior is observed, coupled with fiber extension and straightening crimped fibers. 


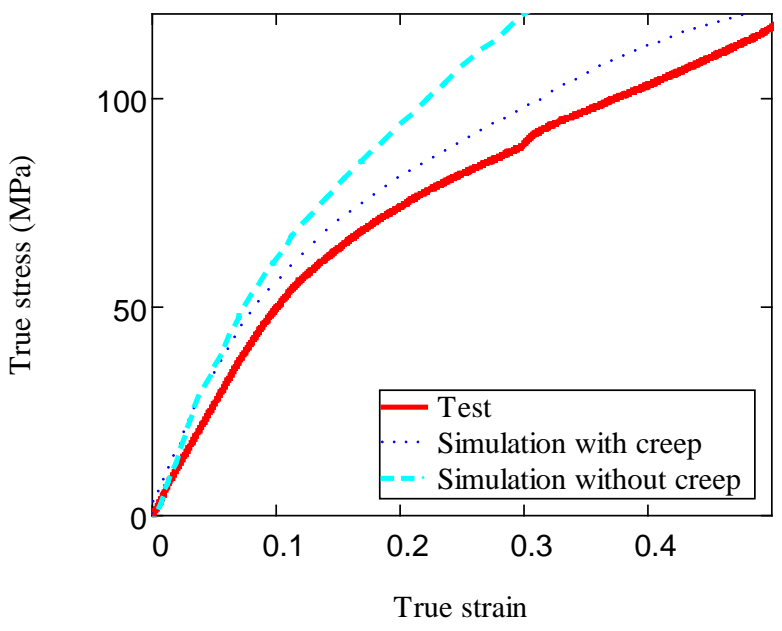

(a)

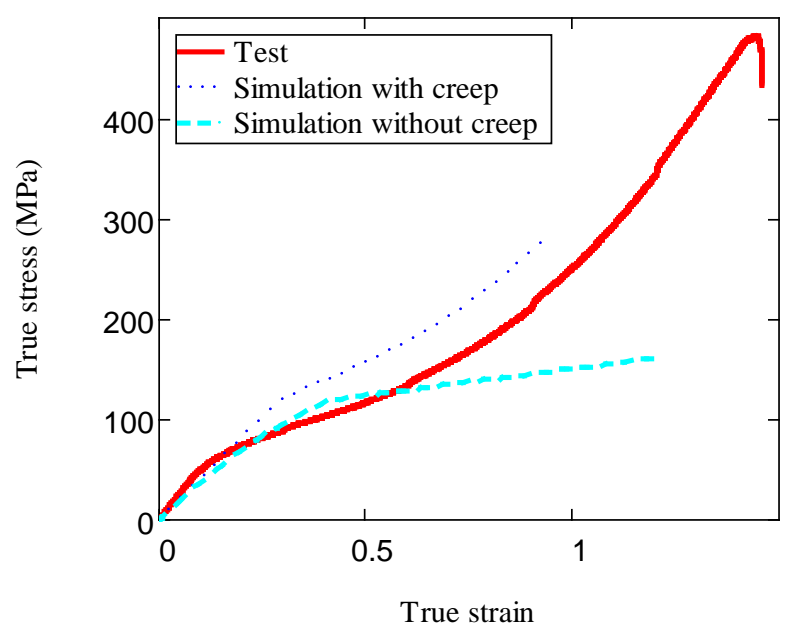

(b)

Fig. 5. True stress - true strain plots of tensile test and simulations with true strain rate of 0.001 1/s: (a) hypoelastic modeling, elastic region; (b) plastic modeling, entire interval

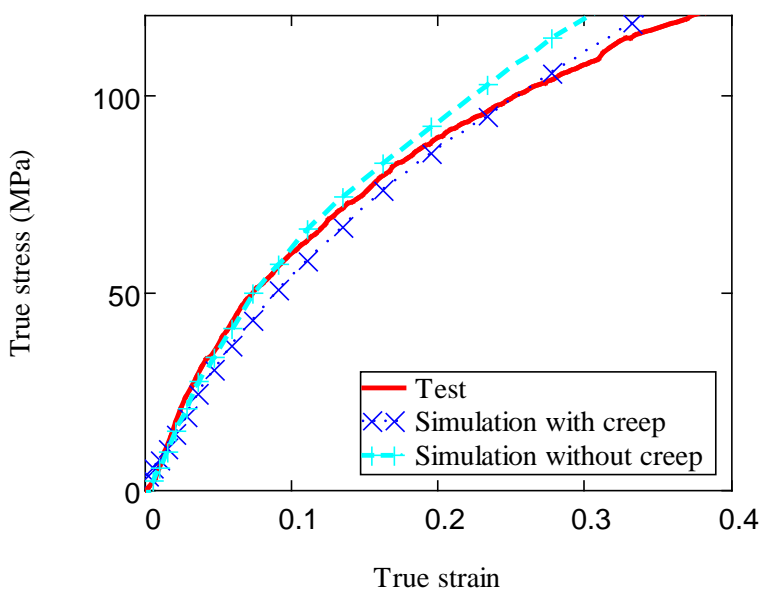

(a)

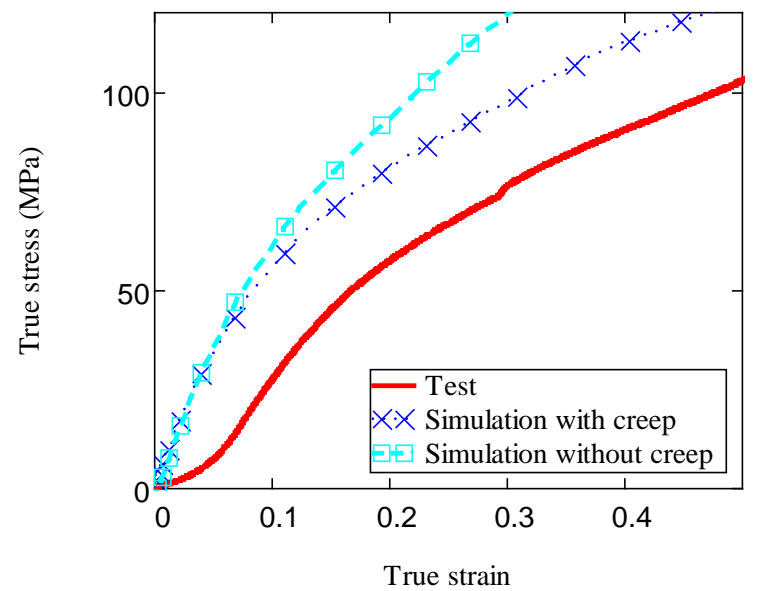

(b)

Fig. 6. True stress - true strain plots of tensile test and simulations with various true strain rates: (a) $0.011 / \mathrm{s}$ : (b) $0.0011 / \mathrm{s}$ (crimped fiber)

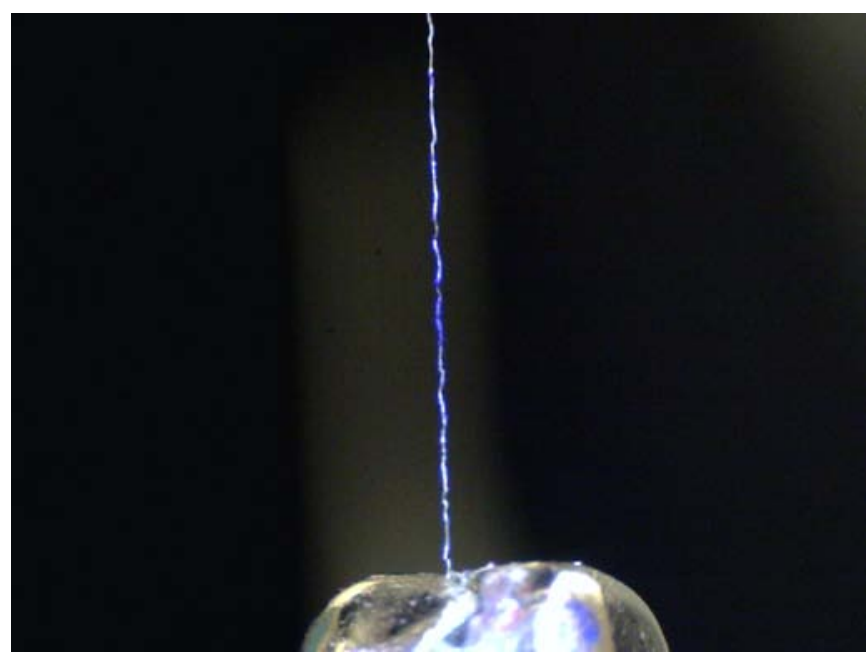

Fig. 7. Fiber sample of the test given in Fig. $6 \mathrm{~b}$ showing the crimp on the specimen 


\section{Conclusions}

In this study, a procedure for determining the viscoelastic behavior of polymeric fibers is presented. Creep tests are performed with different stress levels, and test results are analyzed in order to implement the behavior in the finite element software, MSC Marc Mentat. The tests are carried out for a very short time in order not to avoid the drop of stresses in the first few seconds in contrast to many other studies in the literature. The determined properties are verified and updated by relaxation tests. It was found that the procedure can be used to predict the viscoelastic behavior of polymeric fibers. It is confirmed that the model cannot be used in a plastic range. A different material behavior such as viscoplastic material property should be implemented into the model in this case [8]. Viscoelastic modeling for this material becomes important for the simulation of the tensile tests of tensile strain rate lower than 0.01 1/s. For simulations of tests with higher loading rates, there is no need to implement the viscoelastic behavior. It is important to note that fiber crimp affect the observed material behavior and should be adequately reflected in respective cases.

In addition, the importance of viscoelastic behavior in the first stages of loading is shown in this study. It was expressed that extrapolating the behavior found in the later stages towards the initial stage can produce large errors affecting the behavior of later stages in the simulation as well. In the first stages of loading (while the material is loaded towards the predetermined stress level), both elastic and creep strains increase. As creep strain rate is dependent on the stress, which also increases, it is difficult to separate those types of strain. Thus, the viscoelastic behaviour of initial stage is modified by comparing the relaxation test and simulation results. The procedure described here can be improved for the initial stages of loading.

\section{References}

[1] S.R. Gravelle, K.J. Amberge and D.A. Woodford, Materials \& Design, Vol. 16. (1995), p. 15

[2] A.D. Drozdov and Q. Yuan, International Journal of Solids and Structures, Vol. 40 (2003), p. 2321

[3] Y. C. Bhunavesh and V. B. Grupta, Polymer Vol. 36 (1994), p. 3669

[4] M.J. Dropik , D.H. Johnson and D.E. Roth, 2002 International ANSYS Conference, Penn StateErie, Erie, PA, USA

[5] S.J. Russell: Handbook of Nonwovens (Woodhead Publishing Ltd., England 2007)

[6] X. Hou, Experimental and numerical analysis of deformation of low-density thermally bonded nonwovens, PhD thesis, Loughborough University (2007)

[7] E. Demirci, M. Acar, B. Pourdeyhimi and V. V. Silberschmidt, Anisotropic Elastic-Plastic Mechanical Properties of Thermally Bonded Bicomponent Fibre Nonwovens, $10^{\text {th }}$ Biennial Conference on Engineering Systems Design and Analysis (ESDA), Istanbul, TURKEY (2010), [ISBN: 978-0-7918-3877-8]

[8] A. Ambroziak and P. Klosowski, Task Quarterly, Vol. 12 (2008), p.1001 